\title{
Correlated Transmission Electron Microscopy and Atom Probe Tomography study of Boron distribution in BGaN
}

\author{
Bastien Bonef ${ }^{1}$, Richard Cramer ${ }^{1}$, Feng Wu$^{1}$, and James S. Speck ${ }^{1}$ \\ ${ }^{1}$ Materials Department, University of California, Santa Barbara, CA 93106, USA
}

BGaN ternary alloy has become a promising material in the field of solid state lightning and power electronics. Because of its wide band gap, it could become an alternating material to AlGaN. It has a band gap in the UV spectral region making it a potential candidate for UV laser devices [1] and it can be used as a back barrier in high electron mobility transistors [2]. However, due to the low solubility limit of boron in $\mathrm{GaN}$ and the differences in crystal structure between $\mathrm{BN}$ and $\mathrm{GaN}$, it remains a challenge to grow boron in $\mathrm{GaN}$ and preserve a good crystal quality [3]. Nanometer scale structural characterization are required to optimize the growth conditions of BGaN and to further link its electrical properties to its alloy composition and its structure.

This study deals with a structural and compositional study of BGaN with atom probe tomography (APT) and transmission electron microscopy (TEM). Two samples with boron fractions equals to 0.030 and 0.053 and grown by molecular beam epitaxy (MBE) are investigated. The MBE reactor is a Varian Gen II with a conventional Ga effusion source. B was provided using $\mathrm{BBr}_{3}$ gas. The sample was grown on Ga-face GaN on sapphire templates. TEM cross-sectional samples and APT tips were prepared by dual-beam FIB/SEM by the standard lift-out method. Atomic TEM images were obtained in a FEI Titan microscope operated at $300 \mathrm{kV}$. APT analysis was performed with a Cameca 3000X HR Local Electrode Atom Probe (LEAP) operated in laser-pulse mode.

Atom probe tomography calibration experiments were first performed on $\mathrm{BGaN}$ to find the proper evaporation conditions and avoid detection artifact. These are well known to affect the quantification of nitride semiconductors by APT [4]. We discovered that, when a high field is applied on the APT specimen, boron concentration is over-estimated compared to X-ray diffraction data (XRD). To reduce the applied field on the tip, the laser pulse's power is increased and we found good agreement between boron fractions obtained from APT and XRD. The charge state ratio between $\mathrm{Ga}^{++}$and $\mathrm{Ga}^{+}$ions which give an estimation of the intensity of the field on the surface of the tips can be used to reproduce good evaporation conditions on other BGaN samples.

Results obtained on $\mathrm{B}_{0.030} \mathrm{Ga}_{0.070} \mathrm{~N}$ are presented in figure 1. For such a low boron composition bellow the solubility limit, APT revealed a random distribution of boron in the layer (figure 1(a) and 1(b)). Furthermore, diffraction pattern from TEM analysis revealed that the complete $\mathrm{BGaN}$ layer has a wurzite structure (figure 1(c)). On the opposite, in the $\mathrm{B}_{0.053} \mathrm{Ga}_{0.947} \mathrm{~N}$ sample with boron fraction above the solubility limit, APT revealed a non-random distribution of the III site atoms and the presence of high boron concentration clusters (figure 2(a)). In agreement with APT, TEM images from this sample (figure 2(b)) also revealed the presence of aggregates embed in the wurtzite matrix.

The limitation of TEM for the quantification of low atomic number elements is compensated by the detection efficiency of APT. In this study, we demonstrate the ability of APT to quantify boron in $\mathrm{BGaN}$ layers when evaporation parameters are carefully chosen. Reconstructed volumes reveals a random distribution of boron in $\mathrm{B}_{0.030} \mathrm{Ga}_{0.070} \mathrm{~N}$ but not in $\mathrm{B}_{0.053} \mathrm{Ga}_{0.947} \mathrm{~N}$. APT being unable to resolve atomic planes in BGaN, TEM is employed to verify the integrity of the crystallographic structure. The results obtained from this nanometer scale structural study will allow for the optimization of BGaN growth conditions by molecular beam epitaxy. 


\section{References:}

[1] Sakai, S., Ueta, Y., \& Terauchi, Y. (1993). Japanese journal of applied physics, 32(10R), 4413.

[2] Ravindran, V., Boucherit, M., Soltani, A., Gautier, S., Moudakir, T., Dickerson, J., ... \& Ougazzaden, A. (2012). Applied Physics Letters, 100(24), 243503.

[3] Gautier, S., Patriarche, G., Moudakir, T., Abid, M., Orsal, G., Pantzas, K., ... \& Ougazzaden, A. (2011). Journal of Crystal Growth, 315(1), 288-291.

[4] Rigutti, L., Bonef, B., Speck, J., Tang, F., \& Oliver, R. A. (2017). Scripta Materialia.
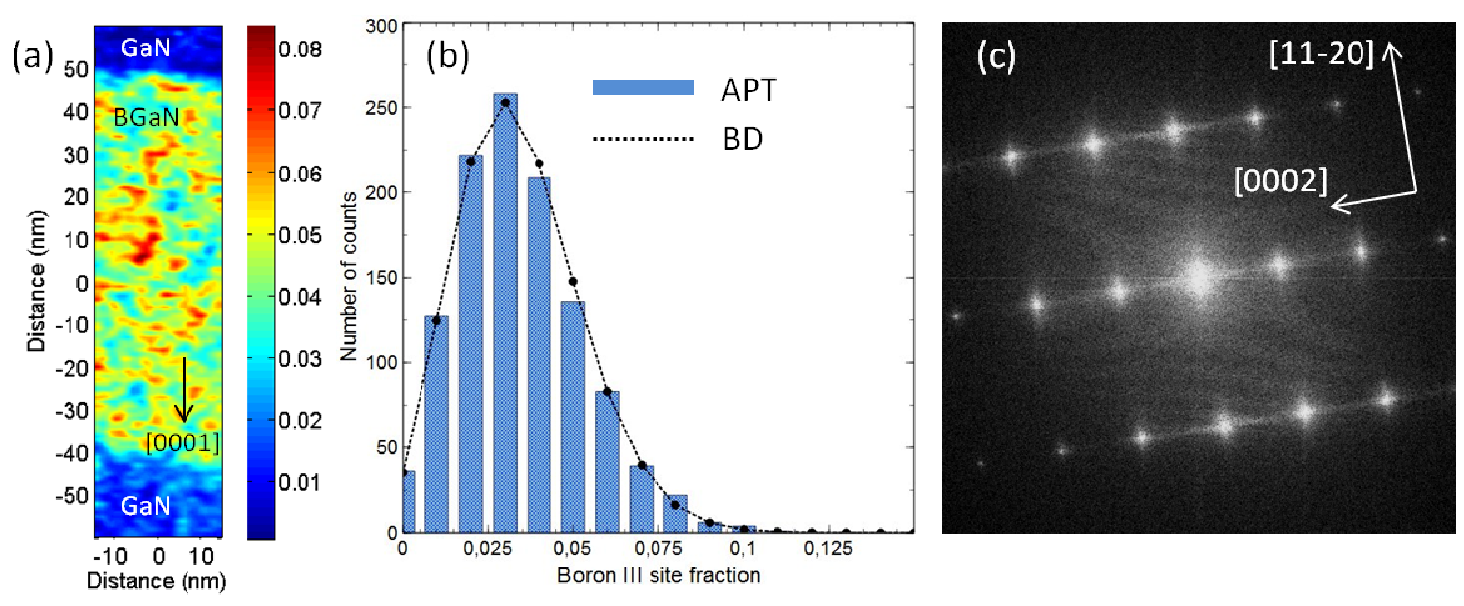

Figure 1. (a) $2 \mathrm{D}$ distribution of boron in the $\mathrm{B}_{0.03} \mathrm{Ga}_{0.07} \mathrm{~N}$ sample obtained from APT; (b) Boron fraction frequency distributions (blue histogram) obtained with APT showing good agreement with the binomial distribution (doted curve). (c) Wurtzite diffraction pattern from the BGaN layer.
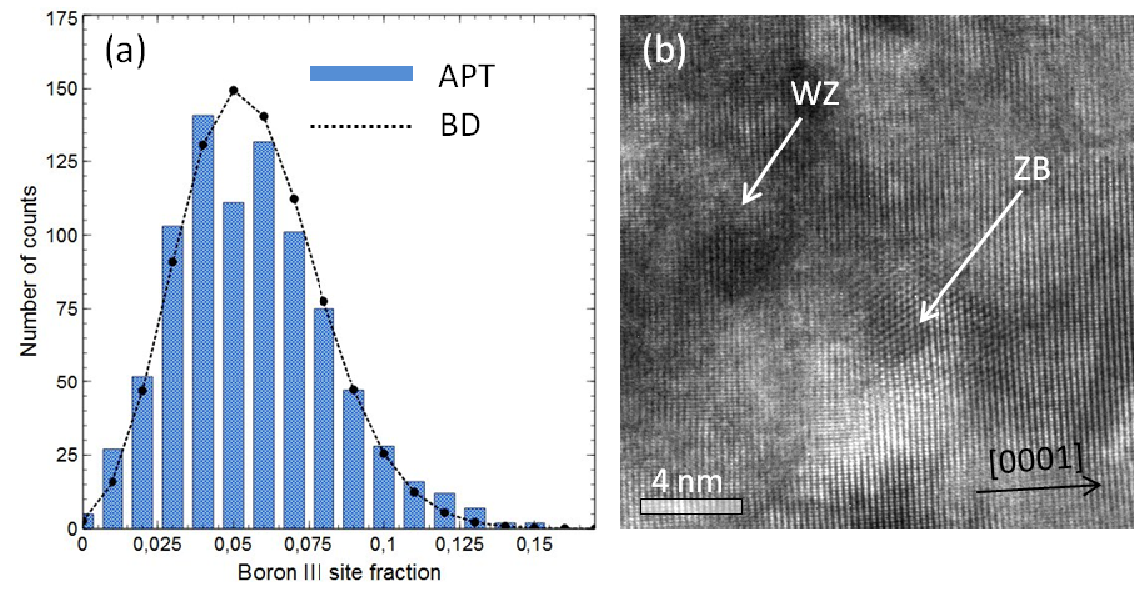

Figure 2. (a) Boron fraction frequency distributions (blue histogram) obtained in the $\mathrm{B}_{0.053} \mathrm{Ga}_{0.947} \mathrm{~N}$ layer showing disparities with the binomial distribution (doted curve). (a) Bright field TEM image of the layer showing an aggregate embed in the wurtzite matrix. 